\title{
Fast, High Resolution Mass Spectrometry Imaging Using a Medipix Pixelated Detector
}

\author{
Julia H. Jungmann, ${ }^{\text {a Luke MacAleese, }}$, Ronald Buijs, ${ }^{\text {a }}$ Frans Giskes, ${ }^{\text {a }}$ \\ Ad de Snaijer, ${ }^{\text {a* }}$ Jan Visser, ${ }^{c}$ Jan Visschers, ${ }^{c}$ Marc J. J. Vrakking, ${ }^{\mathrm{a}, \mathrm{b}}$ and \\ Ron M. A. Heeren ${ }^{\mathrm{a}}$ \\ a FOM Institute for Atomic and Molecular Physics (AMOLF), Amsterdam, The Netherlands \\ ${ }^{\mathrm{b}}$ Max-Born-Institut, Berlin, Germany \\ ${ }^{\mathrm{c}}$ National Institute for Subatomic Physics (Nikhef), Amsterdam, The Netherlands
}

In mass spectrometry imaging, spatial resolution is pushed to its limits with the use of ion microscope mass spectrometric imaging systems. An ion microscope magnifies and then projects the original spatial distribution of ions from a sample surface onto a position-sensitive detector, while retaining time-of-flight mass separation capabilities. Here, a new type of position-sensitive detector based on a chevron microchannel plate stack in combination with a $512 \times 512$ complementary metal-oxide-semiconductor based pixel detector is coupled to an ion microscope. Spatial resolving power better than $6 \mu \mathrm{m}$ is demonstrated by secondary ion mass spectrometry and 8-10 $\mu \mathrm{m}$ spatial resolving power is achieved with laser desorption ionization. A detailed evaluation of key performance criteria such as spatial resolution, acquisition speed, and data handling is presented. (J Am Soc Mass Spectrom 2010, 21, 2023-2030) (C) 2010 Published by Elsevier Inc. on behalf of American Society for Mass Spectrometry

$\mathrm{M}$ ass spectrometry imaging (MSI) [1-3] measurements allow the visualization of the spatial structure and identification of the molecular masses from complex surfaces. High spatial resolution is accomplished with ion-microscope mass spectrometers, where ions are extracted from the sample surface and projected onto a position-sensitive detector. A spatial resolution better than $4 \mu \mathrm{m}$ has been reported using UV/IR laser surface probes in matrix assisted laser desorption ionization (MALDI) [4-8]. A pulsed primary ion beam as a surface probe can achieve higher spatial resolving powers $(1 \mu \mathrm{m})[9,10]$. The spatial resolution can be further improved by using a more focused primary ion/laser desorption ionization surface probe. However, fragmentation of the surface molecules and long measurement times are undesired side effects of decreasing the surface probe area. For instance, at a $2 \times 2 \mu \mathrm{m}$ pixel size $(4 \mu \mathrm{m}$ lateral resolution) and a sample size of $1 \times 1 \mathrm{~mm}$, a typical measurement comprises 250,000 measurement points and can last several hours. An alternate approach to increase the spatial resolution is the use of microscope mode MSI. In the microscope mode, surface molecules are desorbed and ionized over a large sample area, typically $200-300 \mu \mathrm{m}$ in diameter. An ion microscope employs ion optics to project the ionized surface compounds onto a position-sensitive detector while magni-

Address reprint requests to Professor R. M. A. Heeren, FOM Institute for Atomic and Molecular Physics (AMOLF), Science Park 104, 1098 XG Amsterdam, The Netherlands. E-mail: heeren@amolf.nl

* Current address: National Institute for Subatomic Physics (Nikhef), Amsterdam, The Netherlands fying the image and retaining the spatial information defined by the sample surface. With a field of view of $200 \times 200 \mu \mathrm{m}$ and a sample size of $1 \times 1 \mathrm{~mm}, \mathrm{a}$ microscope mode imaging experiment involves 25 measurement points and retains the $4 \mu \mathrm{m}$ lateral resolution given the corresponding ion optical magnification factor. The ion optical magnification allows high-resolution images to be obtained independent of the ionization source. Microscope mode MSI enables fast, highresolution large area imaging provided that an adequate, i.e., fast and position-sensitive, detector is used to record high quality molecular images [4].

Position-sensitive detectors most commonly used for microscope mode MSI are combinations of microchannel plates (MCP), a phosphor screen and a chargecoupled device (CCD) camera [4]. In such an approach, an incident ion is converted into an electron shower by the $\mathrm{MCP}$. The phosphor screen converts the electron shower into photons whose spatial distribution is detected by a CCD camera in the form of an electrical signal/digitized image. MCP-phosphor screen-CCD camera detectors are a reliable and well-established imaging technology. However, the luminescence decay time of the phosphor screen (decay of the intensity from $90 \%$ to $10 \%$ ) amounts to typically hundreds of ns to a few ms. This is slow compared with the obtainable $\mathrm{MCP}$ time resolution (138 ps, in the Amolf setup) and the time resolution required to resolve different $\mathrm{m} / \mathrm{z}$ species on the basis of their time-of-flight towards the detector ( $\sim 15$ ns between $\mathrm{m} / \mathrm{z} 1000$ and 1001, in the Amolf setup). Therefore, mass-resolved imaging is only possible with an MCP-phosphor screen combination if 
prior mass-selection in the ion optics has taken place, or if the MCP is gated at a selected arrival time. Both acquisition modes allow only one mass to be recorded at the same time.

Recently, an MCP stack combined with a delay-linedetector has been implemented in combination with a $\mathrm{C}_{60}{ }^{+}$secondary ion mass spectrometry (SIMS) primary ion beam on an ion microscope for high spatial resolution MSI [11, 12]. The spatial resolving power measured with this setup was $4 \mu \mathrm{m}$. However, substantial signal processing is required to convert the timing signal for every hit on the delay-lines into position information. Subsequently, the image has to be reconstructed, which makes the data collection slow. Furthermore, two-layer delay-line-detectors cannot support large count rates and or do not have multi-hit capabilities for ions hitting the same spot on the detector. While the development of three-layer delay-line-detectors enables the unambiguous detection of two simultaneously arriving particles and the detection of particle showers [13], it does not lift above mentioned limitations inherent to this approach.

CMOS pixel detectors can solve a number of the shortcomings of the present-day imaging detectors for ion microscope MSI and can advance this field of research. Pixel detectors contain thousands of individual channels that can be exploited for parallel detection and are capable of fast measurements. Pixel detectors accommodate large count rates and provide multi-hit capabilities, while the recorded image is the real image and no image reconstruction is needed. Pixel detectors have previously been used for the detection of ions in combination with gas electron multipliers [14, 15]. In combination with an MCP stack, pixel detectors are capable of high-resolution, position-sensitive imaging of ions. They represent a compact, robust, and inexpensive alternative to presently used microscope mode MSI detectors.

Possible future advances based on CMOS pixel detectors are the ease of data acquisition and data processing (direct image acquisition), the high spatial resolution (possibly enhanced by centroiding algorithms), the single-particle counting mode (noise-free particle counting as opposed to charge integration in CCDbased detectors) and the capability of some detector generations to take time-resolved images. In perspective of these attractive CMOS detector features, we describe the in-vacuum implementation and capabilities of a novel type of charged-particle detector-a chevron MCP stack in combination with a Medipix2 imaging readout chip [16, 17] —for microscope mode MSI. Spatial resolution and data handling are key criteria in distinguishing the new detector from approved technologies. Here, standards on transmission electron microscopy (TEM) grids (low mass ions, $\mathrm{m} / \mathrm{z}$ 358) are imaged by secondary ion mass spectrometry (SIMS) and laser desorption ionization (LDI) to characterize the MCP-Medipix2 combination for high spatial resolving power ion-microscope MSI.

\section{Experimental}

\section{The Medipix Detector Family}

The Medipix detector family has been developed within the Medipix collaboration hosted by CERN (Medipix collaboration, www.cern.ch/medipix). The Medipix2 $[16,17]$ application-specific integrated circuit (ASIC) is a hybrid pixel detector with a combined sensor layer and read-out electronics for a compact detector. An array of reverse-biased, fully depleted diodes (typically $300 \mu \mathrm{m}$ of monolithic high-resistivity silicon coated with 150 $\mathrm{nm}-1 \mu \mathrm{m}$ of aluminum) directly converts incident particles into an electron-hole pair current. This electrical signal is processed by the complementary metal oxide semiconductor (CMOS) pixel read-out chip, which works in single particle counting mode (as opposed to charge-integration employed in CCD-based systems). The single particle counting technique, in combination with the pixel detection threshold level, surpresses noise. In principle, the signal-to-noise ratio is infinite given the incident particle's energy is higher than the threshold level energy, which was set above the pixel noise edge. Therefore, the signal-to-noise ratio of the naked Medipix2 detector is independent of $\mathrm{m} / \mathrm{z}$. Every pixel has a 13-bit counter and can accommodate count rates of $\sim 100 \mathrm{kHz}$ for randomly arriving particles. The individual pixel size on the detector is $55 \times 55 \mu \mathrm{m}$ and one chip comprises an array of $256 \times 256$ pixels providing an active area of $\sim 2 \mathrm{~cm}^{2}$. Effectively, one chip contains 65,536 individual detectors, i.e., pixels that can be used for parallel detection. The chips are three-side buttable (one side is reserved for electronic read-out via wire bonds) such that detector arrays of $2 \times 2 n(n=1$, $2,3,4, \ldots)$ chips can be constructed. Here, a Medipix2 quad detector is used, which is a $2 \times 2$ array of Medipix2 chips with an active detector area of $\sim 8 \mathrm{~cm}^{2} \mathrm{~mm}$.

Photons and electrons are directly converted in the detector sensor layer, and can be directly detected provided their energy is high enough. The Medipix detector was primarily conceived as a detector of X-rays and $\boldsymbol{\gamma}$-rays. In earlier work, we have demonstrated the utility of the Medipix detector for imaging photoelectrons with a kinetic energy $>5 \mathrm{keV}$ [18]. Quantum efficiencies of $100 \%$ are expected and have been demonstrated experimentally, with the assumption that the photon/electron energy is fully absorbed with the sensor material [18]. Ions will usually not be accelerated sufficiently to penetrate into the sensor layer. Rather, they are stopped in the $150 \mathrm{~nm}-1 \mu \mathrm{m}$ protective aluminum overlayer that is typically applied on top of the sensor layer. However, ions can be detected indirectly by placement of an MCP in front of the detector [19]. The Medipix detector then registers the electron shower produced by each ion impact on the MCP.

Attractive features of the Medipix chips for mass spectrometry applications are (1) a mass dependence determined only by the MCP that is placed in front of the detector, (2) digitization of the incident signals by 
means of integrated counting circuitry, and (3) a wide dynamic range (13-bit counter per independent pixel). The Medipix detector provides essentially noise-free measurements, since the energy threshold level can be set well above the noise level of the pixels. Additionally, the response of all pixels can be equalized by three software-controllable adjustment bits. The Medipix acquisition software, Pixelman (Pixelman software, version 2008_03_30, http://aladdin.utef.cvut.cz/ofat/others/Pixelman/index.html, Institute of Experimental and Applied Physics, Czech Technical University in Prague, Czech Republic [20], controls parameters such as the acquisition time (from fractions of seconds to hours), the acquisition type (individual frames or data accumulation on the chip) and the file type (text or ASCII file). A data file typically contains a matrix indicating the number of counts registered per individual pixel.

After an acquisition period, the serial chip read-out may last from several $\mathrm{ms}$ to several seconds depending on the size of the chip array $(1 \times 1$ or $2 \times 2 n)$ and the read-out interface. Different read-out interfaces are available for accessing the data generated on the chip [21-23]. Here, a $2 \times 2$ Medipix2 detector is combined with a universal serial bus (USB) read-out interface [21]. This interface board is compact $(75 \times 46 \mathrm{~mm})$ and can directly be coupled to the Medipix printed-circuit board (PCB) without intermediate cabling. The read-out speed is $6 \mathrm{Mbit} / \mathrm{s}$, which yields a read-out time for the entire chip of hundreds of ms. During chip read-out, the pixels cannot accommodate new counts, resulting in a dead time equivalent to the read-out time of the chips. The effect of this read-out/dead time can be minimized by measuring multiple frames, accumulating the images on the chip before read-out and read-out only after a given amount of frames has been measured.

\section{Instrumentation}

In the implementation on our ion microscope (Figure $1 \mathrm{~A})$, the $2 \times 2$ Medipix2 detector is suspended $2.5 \mathrm{~mm}$ behind a $\phi=4 \mathrm{~cm}$ chevron micro-channel plate (MCP). The pores of the MCP have a diameter of $12 \mu \mathrm{m}$ on a 15 $\mu \mathrm{m}$ center-to-center spacing. The MCP front and back sides are biased on voltages of $-2.1 \mathrm{kV}$ and $-0.6 \mathrm{kV}$, respectively. This corresponds to an MCP gain of $8 \times$ $10^{5}$. These settings result in optimal electron shower sizes on the Medipix2 detector with the corresponding optimal spatial resolution. The MCP-Medipix2 detector ensemble, including the USB read-out interface, is mounted in a vacuum chamber.

The Medipix detector read-out electronics out-gasses and is not designed for high vacuum compatibility. Therefore, the detector is operated in a differential pumping geometry (Figure 1B), where the vacuum chamber is constructed in such a way that the MCP represents the conduction limit between the mass spectrometer vacuum system $\left(P=10^{-8}\right.$ Torr $)$ and the vacuum chamber that houses the detector and its asso-
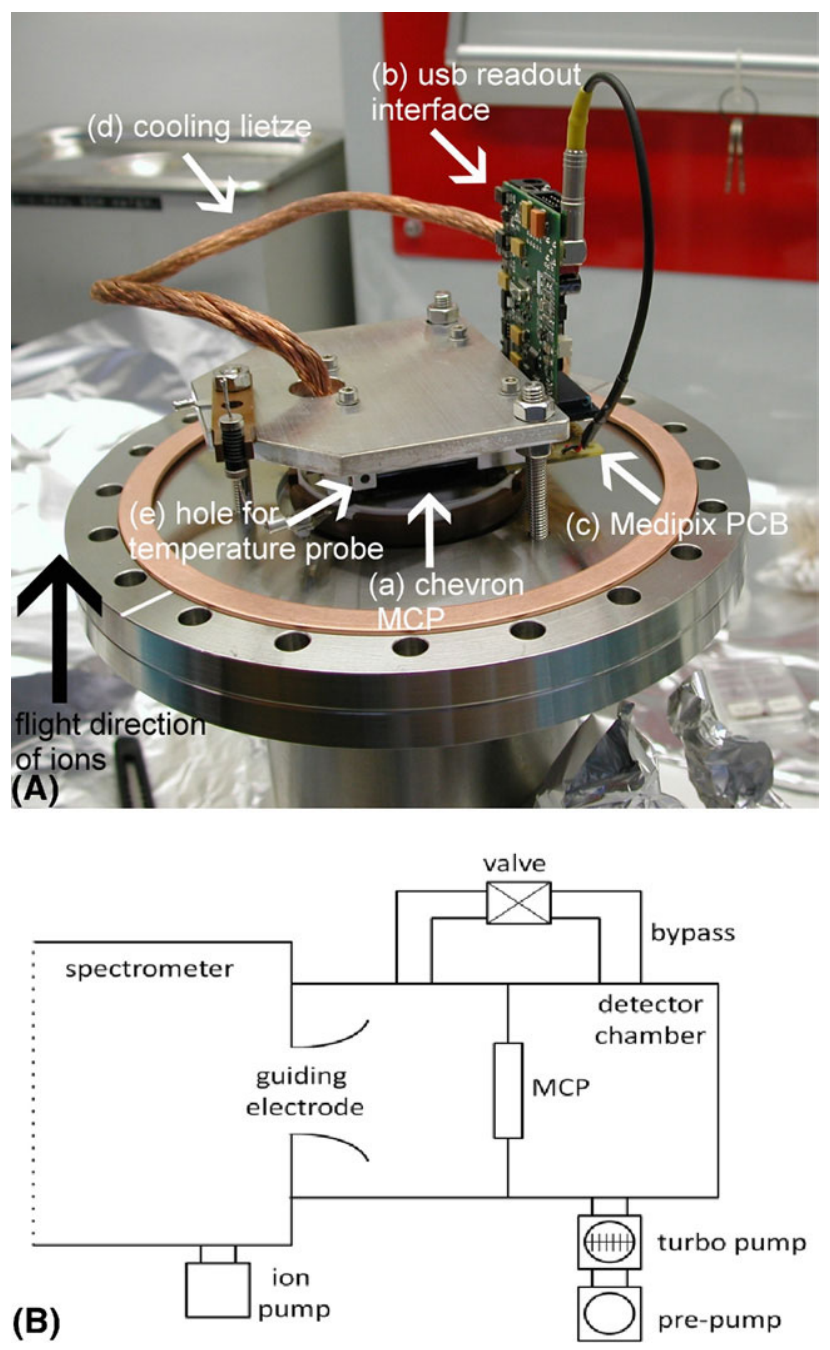

Figure 1. (A) Picture of the detector during assembly. The chevron MCP stack (a) is mounted onto the vacuum flange. The Medipix2 detector is mounted on its Aluminum heat sink at a distance of $2.5 \mathrm{~mm}$ from the MCP. The Medipix2 chips face towards the MCP (down in the picture). The USB read-out interface (b) is connected to the printed-circuit board (c). The copper strand is part of the active cooling system (d); it is clamped to the Medipix heat sink and fed out of the vacuum to the active Peltier element cooling system. The hole in the Medipix heat sink (e) enables the installation of a PT-100 temperature probe, which indicates the heat sink temperature close to the point where the Medipix 2 chip is supported. The black arrow indicates the direction of incident ions arriving from the mass spectrometer on the imaging detector. (B) The differential pumping scheme of the setup. The detector chamber contains the detector and its electronics, which out-gas. The MCP separates the spectrometer and detector vacuum. A bypass between the two chambers protects the MCP from critical pressure gradients during pumping.

ciated electronics ( $P=10^{-7}$ Torr). A bypass between the mass spectrometer vacuum chamber and the detector vacuum chamber protects the MCP from pressure differences during pumping. The power dissipation of the detector and read-out electronics (estimated $5 \mathrm{~W}$ ) requires active cooling and temperature monitoring of the chips in the vacuum. For example, without cooling the detector reaches temperatures above $140{ }^{\circ} \mathrm{C}$ within 45 
min of in-vacuum operation, which is fatal to the detection system and indicates the need for an active cooling system. Therefore, the chips' aluminum heat sink is connected to a $2 \mathrm{~cm}$-diameter copper wire, which is fed to a cooling platform outside the vacuum chamber and cooled by means of Peltier elements. The temperature of the heat sink is monitored by means of a PT-100 temperature probe. Thus, the chips' heat sink temperature can be maintained between 15 and $45^{\circ} \mathrm{C}$.

The MCP + Medipix2 detector system is mounted on a time-of-flight (TOF) ion microscope mass spectrometer (TRIple Focusing Time-of-flight (TRIFT), Physical Electronics, Inc., Chanhassen, MN, USA). The TRIFT mass spectrometer typically delivers an ion optical magnification of a factor of 100 (dependent on the ion optics). Thus, a $55 \times 55 \mu \mathrm{m}$ pixel actually probes an area of $550 \times 550 \mathrm{~nm}$ on the sample surface. However, spatial resolving powers of this order of magnitude are inaccessible with the current setup due to ion optical aberrations [24]. A schematic representation of the setup elements is displayed in Figure 2 and a detailed description of mass spectrometric ion microscopes is covered in detail elsewhere [4].

\section{Experimental Results}

The system was tested with SIMS and LDI. A Cs ${ }^{+}$ion gun with a beam diameter of 270-300 $\mu \mathrm{m}$ was used for SIMS and a Nd:YAG laser (operated at the third harmonic; Bright Solutions, Wedge, model 355; Cura Carpignano, Italy) at a repetition rate of $187 \mathrm{~Hz}$ and a beam diameter of $150-180 \mu \mathrm{m}$ was used for LDI. The mass range is $200<m / z<1850$.

After desorption and ionization from the sample surface, ions traverse the ion-optic and strike the MCP

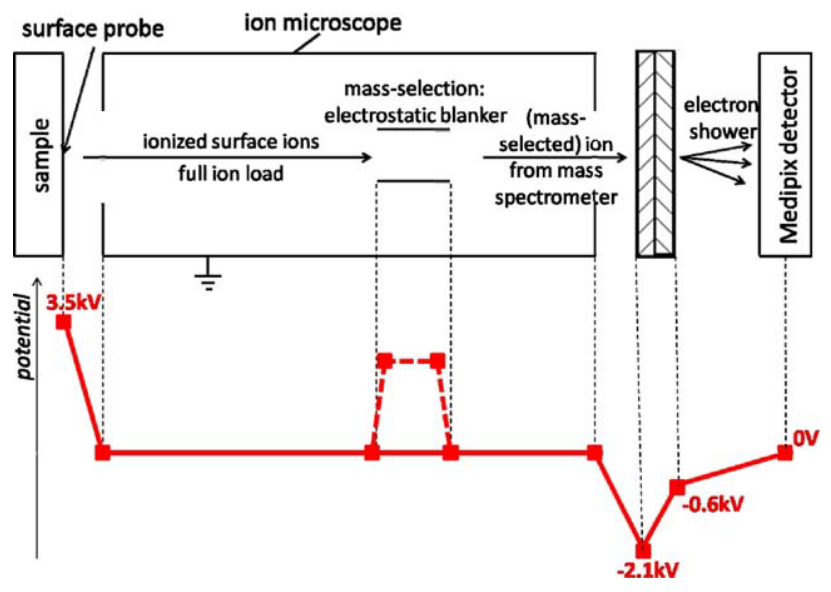

Figure 2. (Top) Schematic representation of the setup elements such as the sample, surface probe, ion microscope, electrostatic blanker, MCP and Medipix2 detector. (Bottom) Potential diagram of the experimental setup. The surface probe desorbs and ionizes the sample surface. The ionized surface particles are electrostatically extracted and guided through the mass spectrometer by the ion optics. Particular $\mathrm{m} / \mathrm{z}$ species can be selected from the full-ion load with the electrostatic blanker. The (mass-selected) ion load is detected by the chevron MCP stack and Medipix2 detector. while retaining their original spatial distribution. The time-of-flight of the ion is related to the $\mathrm{m} / \mathrm{z}$ value [24], and thus single masses can be selected (based on their time-of-flight) by the electrostatic blanker in front of the MCP (Figure 2). This approach has been described in detail elsewhere [4]. Wincadence software (ver. 4.4.0.17; ULVAC-PHI, Inc., Chigasaki, Japan, 2008) is used for instrument control.

Nickel/copper transmission electron microscope (TEM) grids (Agar Scientific, Stanstead, UK) were used to characterize the performance of the Medipix 2 detector on the ion microscope. The TEM grids are hexagonal with pitches of $37 \mu \mathrm{m}$ and bar widths of $8 \mu \mathrm{m}$. The grids are glued (silver paint, Agar G302; Agar Scientific) on top of a layer of green organic dye (green permanent marker, Staedler Lumocolour 318-5) deposited on an indium tin oxide (ITO)-coated glass slide. Figure 3a shows an optical image of a hexagonal TEM grid used in the experiments.

\section{Results and Discussion}

Figure $3 \mathrm{~b}$ displays a total ion image of a hexagonal grid sample generated by positive mode SIMS using the $\mathrm{Cs}^{+}$ surface ionization probe. The field of view is $260 \mu \mathrm{m}$ (the maximum obtainable in this configuration) and the ion optical magnification is 85 . This image is obtained by on-chip integration of $\sim 100$ acquisitions of $0.5 \mathrm{~s}$ each. The chip measures 100 acquisition frames of $0.5 \mathrm{~s}$ each. The data of those frames is meanwhile accumulated on the chip under control of the acquisition software. After 100 frames are acquired, the integrated image is read-out. Alternatively, one could measure 100 individual frames of $0.5 \mathrm{~s}$, read-out every frame after its acquisition, and build the image by off-line integration of the frames. However, this results in substantial read-out and thus dead time. The online image builder of the acquisition software updates the image after each acquired frame. In these measurements, the hexagonal grid structure is clearly resolved by the detector. The image quality does not improve for longer acquisition periods. Typical intensity values of the integrated images range between 200 and 2600 counts per pixel such that the bit depth of $2^{13}$ is not limiting the image acquisition method.

Several characteristic artifacts can be observed in Figure 3. The cross indicates where the four individual chips of the detector are connected and consists of three times larger area edge pixels. Therefore, these pixels display higher count intensities and can be observed in the image. This artifact is inherent to the detector geometry and well-defined such that it can be corrected for. There are fewer signal counts registered in the center of the detector due to a depleted area at the center of the MCP. The image of the grid is slightly distorted due to ion optical distortions which occur especially at a large field of view. An intensity gradient is apparent throughout the image and might be related to sample deterioration. Possibly the intensity gradient is due to a temperature gradient across the chips. The 

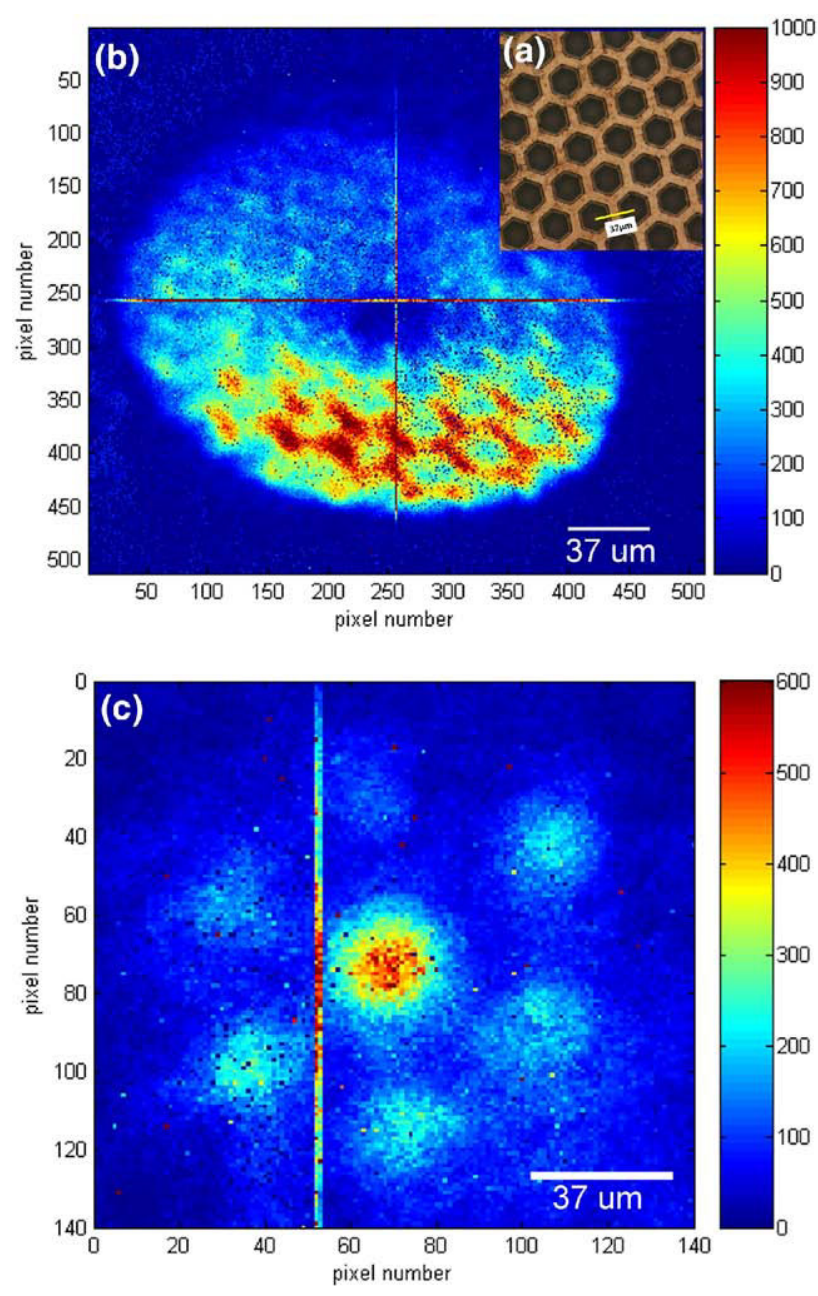

Figure 3. (a) Optical image of a TEM grid. Image as generated with the ion microscope (b) in SIMS mode and (c) in LDI mode, acquired at the same Medipix2 threshold levels. Figure B displays the full $\mathrm{m} / \mathrm{z}$ range (200-1850) obtained by on-chip integration of $\sim 100$ acquisitions of $0.5 \mathrm{~s}$ each. The copper peak is the dominant feature in this experiment. The full $512 \times 512$-pixel image is displayed. (c) shows the mass-selected organic dye $(\mathrm{m} / \mathrm{z}=358$ [25] image which displays a strong signal in the "holes" of the grid. The image is obtained by on-chip integration of $\sim 50$ acquisitions of $0.5 \mathrm{~s}$ each.

copper cooling wire is attached to one side of the chip heat sink and this side is cooled more efficiently than the opposite side, which results in a temperature gradient across the heat sink and chips.

Figure $3 c$ shows part $(140 \times 140$ pixels $)$ of an MSI image of the hexagonal grid sample, which was generated by the laser surface probe (LDI). Here, the hexagonal grid was placed over an organic dye deposited on a glass slide. The mass of the organic dye $(\mathrm{m} / \mathrm{z}=358$ [25] was selected by the electrostatic blanker $(310<\mathrm{m} / \mathrm{z}<$ 410) and shows a strong signal in the "holes" of the hexagonal grid. The field of view is $130 \mu \mathrm{m}$ and the ion optical magnification is 42 . The hexagonal grid structure only covers about one quadrant of the detector surface. Therefore, part of the full detector image, showing the full field of view, is displayed. The small field of view is most likely due to a nonoptimized alignment of the laser beam. The image is generated by on-chip integration of $\sim 50$ acquisitions of $0.5 \mathrm{~s}$ each (one frame of $0.5 \mathrm{~s}$ corresponds to 93 or 94 laser shots since ion generation and detection are not synchronized). The intensity profile which can be observed across the image (higher intensity at the center of the image) is probably due to the laser intensity beam profile. The LDI image uses a large surface probe (laser spot size $\sim 150-180 \mu \mathrm{m}$ ) compared with the spatial resolution obtained in the image. The spatial resolving power in this image (see below) is more than two orders of magnitude better than the surface probe spot size.

Figure $3 b$ and $c$ demonstrate the ability of "complementary" ion detection with this novel detector approach. The figures show complementary images of the metal grid and the organic dye ("holes") obtained in positive mode SIMS and LDI surface ionization mode, respectively.

The spatial resolving power $[2,12]$, i.e., the sharpness sample feature edges, is typically calculated instead of the resolution in terms of pixel size $(55 \times 55 \mu \mathrm{m})$ and ion optical magnification (approximately a factor of 85 in SIMS and a factor of 42 in LDI) resulting in one pixel probing $650 \times 650 \mathrm{~nm}(\mathrm{SIMS})$ and $1.31 \times 1.31 \mu \mathrm{m}$ (LDI) on the sample surface, respectively. The spatial resolving power has been previously defined as the distance between $80 \%$ and $20 \%$ intensity of a feature within the image $[2,12]$. Testing our images against this criterion of resolving power is realized by making a line scan through the image intensity (Figure $4 a$ and $b$ ). We have used the same criteria to evaluate our Medipix generated direct ion images. For that purpose a line scan through the images is made that yields the pixel-topixel intensity properties.

The spatial resolving power of the images shown in Figure $3 b$ and $c$ is evaluated in Figure $4 a$ and $b$. A line scan (black line in the inset of Figure 4a and white line in the inset of Figure $4 \mathrm{~b}$ ) is drawn through features of the image (several hexagonal cells of the grid). Figure 4a displays the signal intensity throughout the sample features (in arbitrary units) versus the spatial distance on the sample (in micrometers) for the SIMS measurement. The horizontal green and red lines indicate the $20 \%$ - and $80 \%$-level of the image features. The spatial resolving power of this measurement is about $5 \mu \mathrm{m}$ (for the high intensity part of the image) at an ion optical magnification of a factor of 85 . A similar analysis of the LDI image (Figure $4 \mathrm{~b}$ ) reveals a spatial resolving power of about 8-10 $\mu \mathrm{m}$ (for the high intensity part of the image) at an ion optical magnification of a factor of 42. The intensity gradient through the LDI images is due to the beam profile of the laser. The resolution of LDI and SIMS scale with the inverse of the ion optical magnification, thus the spatial resolving power obtained with this detector assembly is independent of the surface ionization probe. Figure 4 shows that the detector image matches the physical dimensions of the grid well. 

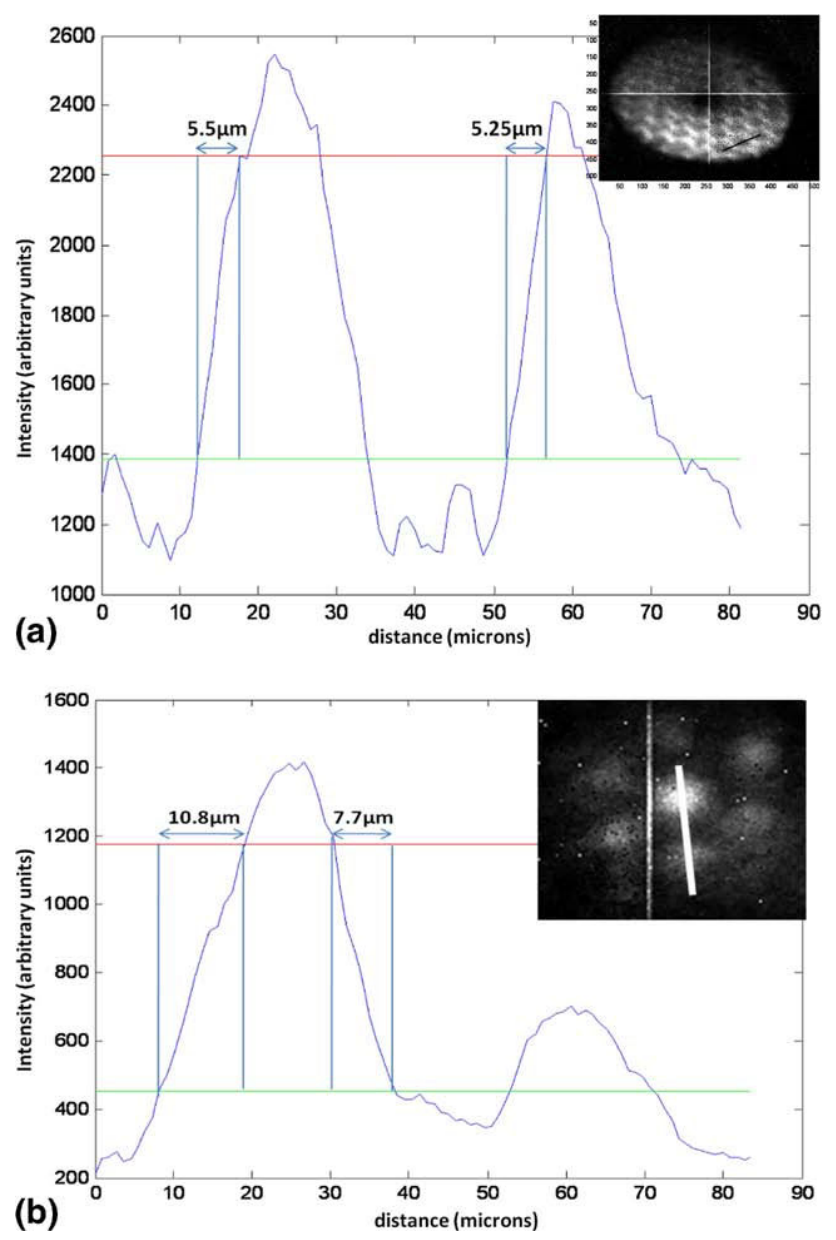

Figure 4. (a) Evaluation of the resolving power the MSI image of Figure 3(b) (inset). The black bar indicates the data over which the resolving power is evaluated. The graph shows a line scan of the intensity per pixel in arbitrary units as a function of the distance on the grid in microns. The graph reveals a resolving power of about $5 \mu \mathrm{m}$. (b) Idem for the LDI image of Figure 3(c).

The on-chip integration of hundreds of individual acquisition frames limits the resolution of the detector. Individual ion hits on the MCP are translated into an electron shower, which typically spans a circle on several pixels on the Medipix2 detector (depending among others on the MCP gain, the distance between the detector and $\mathrm{MCP}$, the voltage across this gap, and the threshold on the Medipix detector). Reconstruction of an image of these "smeared" electron signatures blurs the ion image and limits the spatial resolving power. However, these "cluster" event signatures can be exploited for imaging at sub-pixel spatial resolution by means of centroiding [19].

The dependence of the spatial resolving power on the detection threshold level of the chips was studied to determine optimal operation conditions. The total ion image of a hexagonal grid sample generated by positive mode SIMS and the $\mathrm{Cs}^{+}$surface ionization probe (bottom inset Figure 5) was used to evaluate this relationship. The field of view was $300 \mu \mathrm{m}$ (the maximum obtainable in this configuration) and the ion optical magnification was 60 . The resolving power was evaluated along the yellow line (procedure analogous to the analysis of Figure 4). Figure 5 displays the spatial resolving power in microns versus the increasing detection threshold level in arbitrary units for three peaks along the line scan (red, green, blue data points). The lowest threshold level (number 1) was placed just above the chip intrinsic noise edge. At the highest threshold level (number 10), the features in the figure are just not discriminated away. The threshold level was systematically incremented in ten steps between those two levels. The figure indicates that the spatial resolving power improves from about 10 to about $5 \mu \mathrm{m}$ with an increasing detection threshold. The MSI image insets at threshold level 1, 4, 7, and 10 illustrate how the resolving power improves with higher detection threshold levels. Higher threshold levels cut the image's intensity distribution closer to the top of the distribution, which improves the spatial resolving power as expected. The resulting system resolving power determined here is a convolution of the instrument transmission function and the detector resolving power. As a result, the intrinsic detector resolving power will exceed the values presented in this analysis.

The Medipix detector family will facilitate mosaic mode MSI on biological tissue sections. The current experimental setup is limited in its ability to analyze complex biological samples by limited acceleration voltages. A revision of the setup, including high voltage acceleration capabilities for both ion polarities as well as

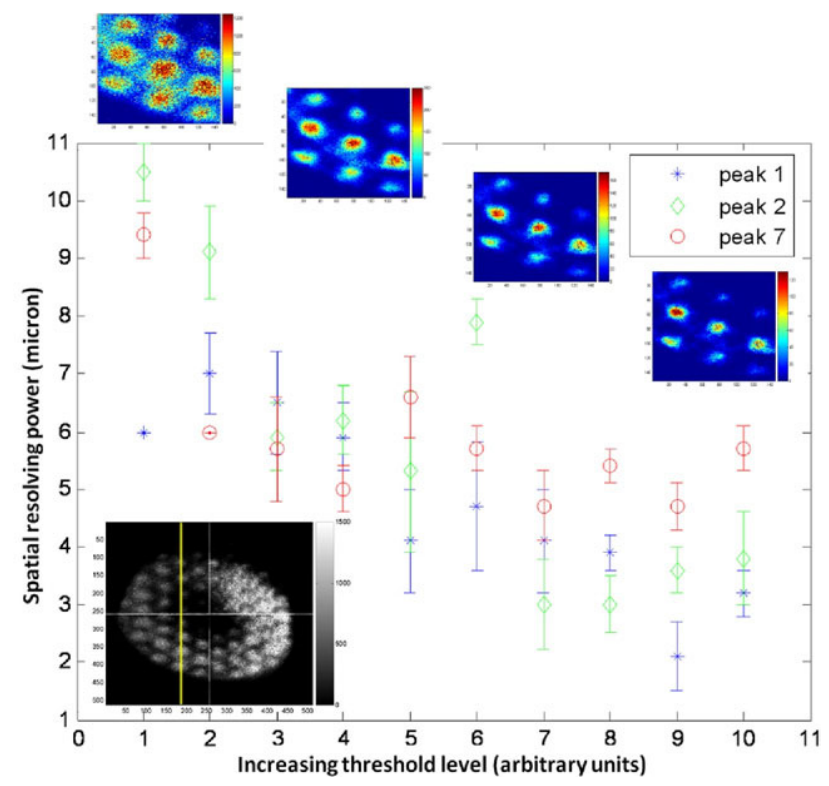

Figure 5. Evaluation of the spatial resolving power as a function of the detection threshold level. The spatial resolving power of an MSI image (bottom inset) is evaluated by the line scan method (yellow line). The spatial resolving power versus the increasing detection threshold level is determined for three peaks along the line scan (red, green, blue). The insets at threshold level 1, 4, 7, and 10 illustrate how the MSI image changes by increasing the chips' threshold level of detection. 
mass-selection based on a Timepix chip, will allow for the analysis of tissue samples by microscope mode imaging mass spectrometry.

\section{Conclusion and Outlook}

We have presented the technical implementation and initial performance of a chevron MCP stack in combination with a Medipix2 ASIC- for direct organic ion imaging using an ion microscope. This detection system has $55 \times 55 \mu \mathrm{m}$ detector pixels and an ion optical magnification of a factor 85 (SIMS) and 42 (LDI) such that an individual pixel probes an area of $650 \times 650 \mathrm{~nm}$ (SIMS) and $1.31 \times 1.31 \mu \mathrm{m}$ (LDI) on the sample surface. The detector is capable of imaging organic material with $\leq 6 \mu \mathrm{m}$ spatial resolving power by positive mode SIMS and with 8-10 $\mu \mathrm{m}$ spatial resolving power using LDI mass spectrometry imaging.

The Medipix detector family has a large potential for imaging mass spectrometry soon. Current imaging detectors provide $2 \mathrm{D}$ imaging capabilities such that the spatial distribution of a particular ion species, i.e., $\mathrm{m} / \mathrm{z}$ value, can only be imaged by selecting the particular ion (based on its time-of-flight) and blanking all other particles. In the presented work, this is achieved with the electrostatic blanker. The implementation of an MCP-Timepix detector assembly will alleviate the need for selective ion blanking. The Timepix detector is a variant of the Medipix2 detector, which, among other features, can measure the time-of-flight of an impinging particle on the pixel level with a time resolution of roughly $10 \mathrm{~ns}$. While the MCP timing will ensure an accurate mass determination of all incoming ions (accurate mass spectra), the Timepix detector images and measures the time-of-flight of different ion species on the pixel level during the same measurement, thereby eliminating the need for mass-selecting specific ions. Thereby, the Timepix detector captures time-resolved images of several $\mathrm{m} / \mathrm{z}$ species in one measurement, which largely facilitates the data taking and reduces the measurement time. The work presented in this paper represents a step in this direction as it demonstrates the in-vacuum implementation of a Medipix2 detector in combination with an $\mathrm{MCP}$ on an ion microscope. Imaging capabilities and data handling are successfully established.

The new detection system combines high spatial resolution imaging with direct imaging capabilities (no image reconstruction/complicated data handling), a mass-independent detector response, and a high dynamic range.

\section{Acknowledgments}

The authors acknowledge that this work is part of the research program of the "Stichting voor Fundamenteel Onderzoek der Materie (FOM)," which is financially supported by the "Nederlandse organisatie voor Wetenschappelijk Onderzoek (NWO)." The authors acknowledge Georg Gademann and Lennaert Klerk
(FOM-Institute Amolf, Amsterdam) for scientific discussion, and the Medipix collaboration hosted by CERN, in particular, our colleagues Marten Bosma, Bas van der Heijden, and Joop Rövekamp (FOM-Institute Nikhef, Amsterdam).

\section{References}

1. Chaurand, P.; Schwartz, S. A.; Caprioli, R. M. Mass Spectrometry Imaging: A New Tool to Investigate the Spatial Organization of Peptides and Proteins in Mammalian Tissue. Curr. Opin. Chem. Biol. 2002, 6, 676-681.

2. McDonnell, L. A.; Heeren, R. M. A. Mass Spectrometry Imaging. Mass Spectrom. Rev. 2007, 26, 606-643.

3. Heeren, R. M. A.; Smith, D. F.; Stauber, J.; Kükrer-Kaletas, B.; MacAleese, L. Mass Spectrometry Imaging: Hype or Hope? J. Am. Soc. Mass Spectrom. 2009, 20, 1006-1014.

4. Luxembourg, S. L.; Mize, T. H.; McDonnell, L. A.; Heeren, R. M. A. High-Spatial Resolution Mass Spectrometric Imaging of Peptide and Protein Distributions on a Surface. Anal. Chem. 2004, 76, 5339-5344.

5. Luxembourg, S. L.; McDonnell, L. A.; Mize, T. H.; Heeren, R. M. A. Infrared Mass Spectrometric Imaging below the Diffraction Limit. J. Proteome Res. 2005, 4(3), 671-673.

6. Spengler, B.; Huber M. Scanning Microprobe Matrix-Assisted Laser Desorption Ionization (SMALDI) Mass Spectrometry: Instrumentation for Sub-Micrometer Resolved LDI and MALDI Surface Analysis. J. Am. Soc. Mass Spectrom. 2002, 13(6), 735-748.

7. Bradshaw, J. A.; Ovchinnikova, O. S.; Meyer, K. A.; Goeringer, D. E. Combined Chemical and Topographic Imaging at Atmospheric Pressure Via Microprobe Laser Desorption/Ionization Mass SpectrometryAtomic Force Microscopy Rapid Commun. Mass Spectrom. 2009, 23, 3781-3786.

8. Koestler, M.; Kirsch, D.; Hester, A.; Leisner, A.; Guenther, S.; Spengler, B. A High-Resolution Scanning Microprobe Matrix-Assisted Laser Desorption/Ionization Ion Source for Imaging Analysis on an Ion Trap/ Fourier Transform Ion Cyclotron Resonance Mass Spectrometer. Mass Spectrom. 2008, 22, 3275-3285.

9. Veryovkin, I. V.; Calaway, W. F.; Tripa, C. E.; Pellin, M. J. Mass Spectrometry on the Nanoscale with Ion Sputtering Based Techniques: What is Feasible. Nucl. Instrum. Methods B 2007, 261, 508-511.

10. Ghosal, S.; Fallon, S. J.; Leighton, T. J.; Wheeler, K. E.; Kristo, M. J.; Hutcheon, I. D.; Weber, P. K. Imaging and 3D Elemental Characterization of Intact Bacterial Spores by High-Resolution Secondary Ion Mass Spectrometry. Anal. Chem. 2008, 80, 5986-5992.

11. Froesch, M.; Luxembourg, S. L.; Verheijde, D.; Heeren, R. M. A. Mass Spectrometry Imaging Using a Delay-Line Detector. Eur. J. Mass Spectrom. 2010, 16, 35-45.

12. Klerk, L. A.; Lockyer, N. P.; Kharchenko, A.; MacAleese, L.; Dankers, P. Y. W.; Vickerman, J. C.; Heeren, R. M. A. $\mathrm{C}_{60}{ }^{+}$Secondary Ion Microscopy Using a Delay Line Detector. Anal. Chem. 2010, 82(3), 801-807.

13. Jagutzki, O.; Cerezo, A.; Czasch, A.; Dorner, R.; Hattas, M.; Min Huang; Mergel, V.; Spillmann, U.; Ullmann-Pfleger, K.; Weber, T.; SchmidtBocking, H.; Smith, G. D. W. Multiple Hit Readout of a Microchannel Plate Detector with a Three-Layer Delay-Line Anode. IEEE Trans. Nucl. Sci. 2002, 49(5), 2477-2483.

14. Colas, P.; Colijn, A. P.; Fornaini, A.; Giomataris, Y.; Van der Graaf, H.; Heijne, E. H. M.; Llopart, X.; Schmitz, J.; Timmermans, J.; Visschers, J. L. The Readout of a GEM or Micromegas-Equipped TPC by Means of the Medipix2 CMOS Sensor as Direct Anode. Nucl. Instrum. Methods A 2004, $1 / 2,506-510$

15. Bamberger, A.; Desch, K.; Renz, U.; Titov, M.; Vlasov, N.; Wienemann, P.; Zwerger, A. Resolution Studies on $5 \mathrm{GeV}$ Electron Tracks Observed with Triple-GEM and MediPix2/TimePix-Readout. Nucl. Instrum. Methods A 2007, 581, 274-278.

16. Llopart, X.; Campbell, M.; Dinapoli, R.; San Segundo, D.; Pernigotti, E. Medipix2, a 64 k Pixel Readout Chip with 55 Micron Square Elements Working in Single Photon Counting Mode IEEE Trans. Nucl. Sci. 2002, 49, 2279-2283.

17. Llopart, X.; Campbell, M. First Test Measurements of a $64 \mathrm{k}$ Pixel Readout Chip Working in Single Photon Counting Mode. Nucl. Instrum. Methods A 2003, 509, 157-163.

18. Gademann, G.; Huismans, Y.; Gijsbertsen, A.; Jungmann, J.; Visschers, J.; Vrakking, M. J. J. Velocity Map Imaging Using an In-Vacuum Pixel Detector. Rev. Sci. Instrum. 2009, 8, 103105-103107.

19. Vallerga, J.; McPhate, J.; Tremsin, A.; Siegmund, O.; Mikulec, B.; Clark, A. Optically Sensitive Medipix2 Detector for Adaptive Optics Wavefront Sensing. Nucl. Instrum. Methods A 2005, 546, 263-269.

20. Holy, T.; Jakubek, J.; Pospisil, S.; Uher, J.; Vavrik, D.; Vykydal, Z. Data Acquisition and Processing Software Package for Medipix2. Nucl. Instrum. Methods A 2006, 1, 254-258.

21. Vykydal, Z.; Jakubek, J.; Pospisil, S. A USB Interface for Medipix2 Pixel Device Enabling Energy and Position-Sensitive Detection of Heavy Charged Particles. Nucl. Instrum. Methods A 2006, 563, 112-115.

22. (a) San Segundo Bello, D; van Beuzekom, M.; Jansweijer, P.; Verkooijen, H.; Visschers, J. An Interface Board for the Control and Data Acquisition of the Medipix2 chip. Nucl. Instrum. Methods A 2003, 509, 164-170. 
23. (b) Vykydal, Z.; Visschers, J.; Sabuncuoglu. Tezcan, D.; De. Munck, K.; Borgers, T.; Ruythooren, W.; De. Moor, P. The RELAXd project: Development of Four-Side Tilable Photon-Counting Imagers. Nucl. Instrum. Methods A 2008, 591, 241-244.
24. Schueler, B. W. Microscope Imaging by Time-of-Flight Secondary Ion Mass Spectrometry. Microsc. Microanal. M. 1992, 3, 11913.

25. Klerk, L. A. Mass Spectrometry Imaging of Polymeric Materials. Ph.D. Thesis, University of Utrecht, 2009; p. 85. 